\title{
Avaliação de Níveis de Fósforo na Dieta de Novilhos Nelore em Crescimento: Efeitos no Desempenho
}

\author{
Maria Luiza Franceschi Nicodemo1, Sheila da Silva Moraes', Ivan Valadão Rosa², Manuel \\ Cláudio Motta Macedo', Luiz Roberto Lopes de S. Thiago', Cláudio Ribeiro dos Anjos ${ }^{3}$
}

\begin{abstract}
RESUMO - A adequação das recomendações de fósforo para novilhos Nelore em crescimento foi avaliada por meio do ganho de peso, do consumo e da conversão alimentar do alimento durante 250 dias. Quinze novilhos receberam dieta basal deficiente em P, adequada nos demais nutrientes, capaz de proporcionar ganhos de $500 \mathrm{~g} / \mathrm{d}$. A dieta foi suplementada (tratamento $1=15 \mathrm{~g}$ P/dia e tratamento $2=11 \mathrm{~g} \mathrm{P} /$ dia) ou não (tratamento $3=5 \mathrm{~g}$ P/dia) com fosfato monoamônico. A deficiência de $\mathrm{P}$ não afetou as digestibilidades in vivo da matéria orgânica medida aos 60 dias e ao final do experimento, nem a digestibilidade da matéria seca ao final do experimento. O consumo médio de matéria seca foi de 5,56; 5,28; e 3,69 kg/dia, nos tratamentos 1,2 e 3, respectivamente. A deficiência de $\mathrm{P}$ provocou quedas no consumo, no ganho de peso e na conversão alimentar. Os novilhos de $300 \mathrm{~kg}$ alimentados com $11 \mathrm{~g}$ P/dia obtiveram ganhos de 500$600 \mathrm{~g} / \mathrm{dia}$, sem apresentar sinais de deficiência.
\end{abstract}

Palavras-chave: bovino de corte, conversão alimentar, ganho de peso, exigências nutricionais

\section{Evaluation of Phosphorus Levels in the Diet of Growing Nellore Steers: Effects on the Performance}

\begin{abstract}
The adequacy of the recommendation of phosphorus for growing Nellore steers was evaluated by means of the weight gain, feed intake and feed:gain ratio during 250 days. Fifteen steers were fed a basal P-deficient but otherwise adequate in the other nutrients, enabling gains of $500 \mathrm{~g} / \mathrm{day}$. The diet was supplemented (treatment $1=15 \mathrm{~g} \mathrm{P} / \mathrm{day}$, treatment $2=11 \mathrm{~g} \mathrm{P} / \mathrm{day}$ ) or not (treatment $3=5 \mathrm{~g}$ P/day) with monoammonium phosphate. Phosphorus deficiency did not affect organic matter in vivo digestibilities determined at 60 days and at the end of the experiment, nor dry matter digestibility at the end of the experiment. Average dry matter intakes were 5.56, 5.28 and $3.69 \mathrm{~kg} / \mathrm{day}$, for treatments 1, 2 and 3, respectively. Feed intake, weight gain and feed:gain ratio were reduced by phosphorus deficiency. Steers fed $11 \mathrm{~g}$ of P/day gained 500-600 g/day without signs of deficiency.
\end{abstract}

Key words: beef cattle, feed: gain, nutrient requirement, weight gain

\section{Introdução}

O fósforo, geralmente, é deficiente na dieta de gado de corte a pasto (TOKARNIA et al., 1988). A deficiência de fósforo está associada a uma série de sintomas inespecíficos, como baixo ganho de peso, consumo reduzido, depravação de apetite e falha reprodutiva (UNDERWOOD, 1981). As concentrações de fósforo indicadas para dietas de bovinos de corte foram aparentemente superestimadas em algumas situações (CALL et al., 1978, 1986; LITTLE, 1980; MARGON et al., 1982; e EZEQUIEL et al., 1988). Comparando as recomendações do NATIONAL RESEARCH COUNCIL - NRC de 1984 e 1996, nota-se que os requisitos para mantença foram reduzidos em cerca de $30 \%$, enquanto para ganho de peso foram aumentados (devido à redução do coeficiente de absorção utilizado $[0,68$ vs 0,85$])$ na versão mais recente.

Experimentos de alimentação são úteis na avaliação de requisitos nutricionais calculados pelo método fatorial. Parâmetros produtivos podem ser utilizados nessa avaliação; nesse caso, os níveis que permitem máxima eficiência de produção indicariam o suprimento adequado do nutriente em questão. O objetivo deste estudo foi avaliar a adequação das recomendações de exigências nutricionais para novilhos Nelore em crescimento quanto à produção de carne e ao consumo e à utilização da matéria seca.

\footnotetext{
1 Pesquisador da Embrapa Gado de Corte. Caixa Postal 154, CEP 79002-070, Campo Grande, MS

2 Médico-veterinário.

${ }^{3}$ Assistente de Pesquisa da Embrapa Agropecuária Oeste.
} 


\section{Material e Métodos}

Quinze novilhos Nelore de sobreano pesando cerca de $215 \mathrm{~kg}$ foram tratados contra parasitas e alojados em baias individuais. No começo do experimento, foram divididos de acordo com o peso em três grupos (cinco novilhos por grupo) e receberam uma dieta basal (Tabela 1) suplementada ou não com fosfato monoamônico. O volumoso (palha e sabugo de milho triturados) participou com 30-35\% da ração. A dieta foi calculada usando as recomendações do NRC (1984), sendo adequada em todos os nutrientes, exceto $P$, e atendia a 110,80 e $50 \%$ dos requisitos de fósforo nos tratamentos 1,2 e 3 , respectivamente. A inclusão de fosfato monoamônico (contendo cerca de $9,5 \%$ de N) na ração dos tratamentos 1 e 2 não constitui aporte significativo de proteína à dieta. A dieta foi ajustada periodicamente em função do peso vivo e fornecida em quantidades capazes de proporcionar ganhos de peso da ordem de $600 \mathrm{~g} / \mathrm{dia}$, em duas porções diárias, às 8 e $16 \mathrm{~h}$. $\mathrm{O}$ consumo individual foi medido diariamente e amostras do alimento oferecido $(5 \%)$ e rejeitado $(10 \%)$, retidas para análise. A água foi suprida por meio de bebedouros automáticos. Ingestão de alimento foi também corrigida para tamanho metabólico (matéria seca, $\mathrm{g} /$ peso vivo ${ }^{0,75}$ ) para remover qualquer efeito de diferenças de peso vivo da comparação entre os tratamentos. Os animais foram pesados a cada 15 dias. Os novilhos foram dotados de bolsas para coleta total de fezes aos 60 e 243 dias de experimentação, e amostras foram retidas para análise. Os dois ensaios de digestibilidade tiveram duração de sete dias. Foram analisados os teores de matéria seca e matéria orgânica (HARRIS, 1970) no alimento e nas fezes. Análises complementares no pool mensal/tratamento incluíam fibra detergente neutro e digestibilidade in vitro da matéria orgânica (HARRIS, 1970), assim como cálcio e fósforo (MORAES e RABELO, 1986). As análises estatísticas foram realizadas por intermédio de análise de variância (ANOVA e GLM) e regressão, conforme procedimento do aplicativo SAS (SAS, 1985).

\section{Resultados e Discussão}

Os animais dos tratamentos 1 e 2 tiveram ganhos de peso semelhantes, da ordem de 500-600 g/dia (Tabela 2), enquanto os animais do tratamento 3 ganharam apenas $200 \mathrm{~g} /$ dia. A diferença já se manifestou aos 71 dias de experimentação $(\mathrm{P}<0,01$, Figura 1). A ingestão de matéria seca neste tratamen- to foi cerca de $30 \%$ mais baixa (Tabela 3) e, certamente, contribuiu para a queda no ganho de peso. Nota-se que o consumo de matéria seca, em função do peso metabólico (Tabela 4), foi constante nos tratamentos 1 e 2 , mas diminuiu significativamente $(\mathrm{P}<0,001)$ com o tempo no tratamento 3 .

Tabela 1 - Composição média da ração basal

Table 1 - Average composition of the basal diet

\begin{tabular}{|c|c|}
\hline $\begin{array}{l}\text { Composição } \\
\text { Composition }\end{array}$ & $\%$ \\
\hline $\begin{array}{l}\text { Uréia } \\
\text { Urea }\end{array}$ & 2,209 \\
\hline $\begin{array}{l}\text { Raiz de mandioca dessecada } \\
\text { Cassava meal }\end{array}$ & 71,790 \\
\hline $\begin{array}{l}\text { Melaço em pó } \\
\text { Dried molasses }\end{array}$ & 15,311 \\
\hline $\begin{array}{l}\text { Farinha de sangue } \\
\text { Blood meal }\end{array}$ & 0,906 \\
\hline $\begin{array}{l}\text { Farelo de soja } \\
\text { Soybean meal }\end{array}$ & 1,811 \\
\hline $\begin{array}{l}\text { Farelo de amendoim } \\
\text { Peanut meal }\end{array}$ & 7,038 \\
\hline $\begin{array}{l}\text { Mistura mineral } \\
\text { Mineral mixture }\end{array}$ & 0,935 \\
\hline
\end{tabular}

Composição média da mistura mineral (Average mineral mixture composition) (\%): $\mathrm{NaCl}=48,433 ; \mathrm{KIO}_{3}=0,014 ; \mathrm{CuSO}_{4}=0,702 ; \mathrm{CoSO}_{4}=0,028$; $\mathrm{ZnSO}_{4}=3,156 ; \mathrm{Na}_{2} \mathrm{SeO}_{3}=0,007 ; \mathrm{S}^{\circ}=9,512 \mathrm{e} \mathrm{CaCo}_{3}=38,148$.

Tabela 2 - Equações de regressão linear do peso vivo (PV) em função do tempo (dias, d)

Table 2 - Linear regression equations on live weight ( $L W$ ) and time (days,d)

\begin{tabular}{lccc}
\hline $\begin{array}{l}\text { Tratamento } \\
\text { Treatment }\end{array}$ & $\begin{array}{l}\text { Regressão } \\
\text { Regression }\end{array}$ & Anova & $\mathrm{r}^{2}$ \\
\hline 1 & $\mathrm{PV}=222,78+0,605 \mathrm{~d}$ & $\mathrm{P}<0,001$ & 0,92 \\
2 & $\mathrm{PV}=217,17+0,533 \mathrm{~d}$ & $\mathrm{P}<0,001$ & 0,90 \\
3 & $\mathrm{PV}=229,76+0,231 \mathrm{~d}$ & $\mathrm{P}<0,001$ & 0,61 \\
\hline
\end{tabular}

Tabela 3 - Consumo diário de matéria seca, fósforo e cálcio

Table 3 - Daily intake of dry matter, phosphorus and calcium

\begin{tabular}{lccr}
\hline Item & \multicolumn{3}{c}{$\begin{array}{c}\text { Tratamento } \\
\text { Treatment }\end{array}$} \\
\cline { 2 - 4 } & 1 & 2 & 3 \\
\hline Matéria seca, kg & $5,56^{\mathrm{a}}$ & $5,28^{\mathrm{a}}$ & $3,69^{\mathrm{b}}$ \\
Dry matter & & & \\
$\mathrm{P}, \mathrm{g}$ & $14,65^{\mathrm{a}}$ & $10,75^{\mathrm{b}}$ & $4,83^{\mathrm{c}}$ \\
$\mathrm{Ca}, \mathrm{g}$ & $38,76^{\mathrm{a}}$ & $37,51^{\mathrm{a}}$ & $27,55^{\mathrm{b}}$ \\
$\mathrm{Ca} / \mathrm{P}$ & $2,64^{\mathrm{b}}$ & $3,50^{\mathrm{b}}$ & $5,87^{\mathrm{a}}$ \\
\hline
\end{tabular}

Médias, na linha, seguidas por diferentes letras são diferentes $(\mathrm{P}<0,05)$ pelo teste Tukey.

Means, within a row, followed by different letters are different $(P<.05)$ by Tukey test. 
Rev. bras. zootec.

Tabela 4 - Equações de regressão linear do consumo de matéria seca $\left(\mathrm{MS}_{1}, \mathrm{~kg} / \mathrm{dia}\right.$ ou $\left.\mathrm{MS}_{2}, \mathrm{~g} / \mathrm{kg}^{0,75} / \mathrm{dia}\right)$ em função do tempo (dias, d) nas 8 primeiras semanas de experimentação

Table 4 - Linear regression equations dry matter intake $\left(M S_{1}\right.$, $\mathrm{kg} /$ day or $\mathrm{MS}, \mathrm{g} / \mathrm{kg}^{0.75} /$ day) on time (days, d) in the first 8 weeks of trial

\begin{tabular}{llll}
\hline $\begin{array}{l}\text { Tratamento } \\
\text { Treatment }\end{array}$ & \multicolumn{1}{c}{$\begin{array}{c}\text { Regressão } \\
\text { Regression }\end{array}$} & Anova & $\mathrm{r}^{2}$ \\
\hline 1 & $\mathrm{MS}_{1}=4,72+0,012 \mathrm{~d}$ & $\mathrm{P}<0,001$ & 0,42 \\
2 & $\mathrm{MS}_{1}=4,53+0,013 \mathrm{~d}$ & $\mathrm{P}<0,01$ & 0,21 \\
3 & $\mathrm{MS}_{1}=5,06-0,020 \mathrm{~d}$ & $\mathrm{P}<0,01$ & 0,21 \\
\hline 1 & $\mathrm{MS}_{2}=84,2-0,013 \mathrm{~d}$ & $\mathrm{P}>0,05$ & 0,002 \\
2 & $\mathrm{MS}_{2}=81,3+0,051 \mathrm{~d}$ & $\mathrm{P}>0,05$ & 0,01 \\
3 & $\mathrm{MS}_{2}=89,2-0,510 \mathrm{~d}$ & $\mathrm{P}<0,001$ & 0,44 \\
\hline
\end{tabular}

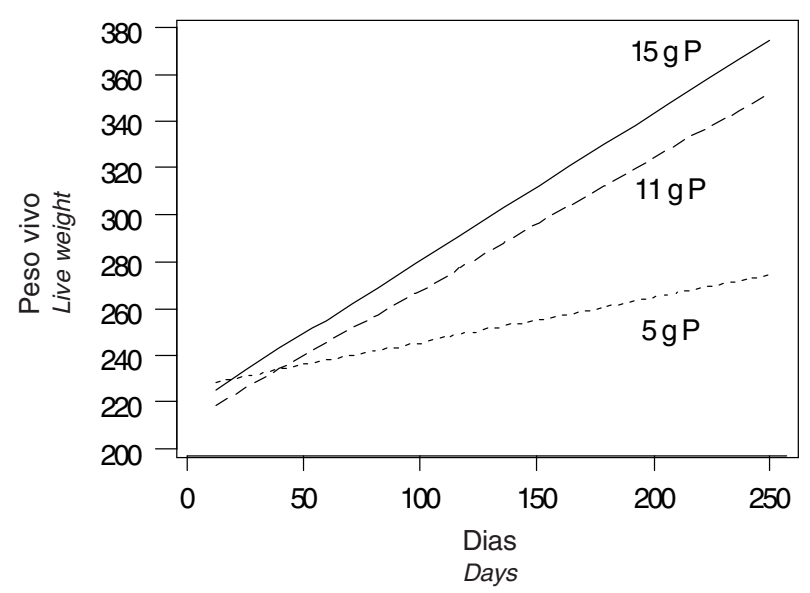

Figura 1 - Evolução do peso vivo (kg) em novilhos recebendo dietas com 15, 11 e $5 \mathrm{~g} \mathrm{P} /$ dia.

Figure 1 - Live weight $(\mathrm{kg})$ increment for steers fed diets with 15 , 11 and $5 \mathrm{~g} P /$ day.

A redução no consumo de alimento é um dos principais sintomas da deficiência de $\mathrm{P}$, e dois mecanismos fisiológicos têm sido estudados: a interferência da deficiência de P no metabolismo intermediário e a redução na atividade microbiana.

As digestibilidades da matéria seca e matéria orgânica (medidas in vivo aos 243 dias) não foram afetadas negativamente pelo baixo nível de P na dieta (Tabela 5), confirmando observações de BORTOLUSSI et al. (1996). Apenas a digestibilidade da matéria seca aos 60 dias de experimento mostrou efeito dos níveis de $\mathrm{P}(\mathrm{P}<0,01)$. O aumento da digestibilidade no tratamento 3 provavelmente deveu-se ao maior tempo propiciado à ação dos microorganismos (menor taxa de passa-
Tabela 5 - Equações de regressão linear da digestibilidade in vivo da matéria seca (DIVMS) e da matéria orgânica (DIVMO), em função do nível de $P$ na dieta $(P)$

Table 5 - Linear regression equations on dry matter and organic matter in vivo disappearance (DMIVD and OMIVD, respectively) on $P$ concentration in the diet $(P)$

\begin{tabular}{llll}
\hline $\begin{array}{l}\text { Dias de experimentação } \\
\text { Days of trial }\end{array}$ & $\begin{array}{l}\text { Regressão } \\
\text { Regression }\end{array}$ & Anova & $\mathrm{r}^{2}$ \\
\hline 60 & DIVMS=72,57-0,602P & $\mathrm{P}<0,01$ & 0,066 \\
243 & DIVMS=71,38-0,169P & $\mathrm{P}>0,05$ & 0,011 \\
\hline 60 & DIVMO=72,13-0,253P & $\mathrm{P}>0,05$ & 0,013 \\
243 & DIVMO=77,43-0,204P & $\mathrm{P}>0,05$ & 0,022 \\
\hline
\end{tabular}

gem), quando há menor consumo de alimento (TERNOUTH e SEVILLA, 1990).

Os microorganismos do rúmen parecem ser menos sensíveis que o hospedeiro à deficiência de $\mathrm{P}$. Esta resistência se deve, provavelmente, à eficiente reciclagem do elemento por meio da saliva e aos requisitos relativamente baixos dos microorganismos (NEL e MOIR, 1974; DURAND e KAWASHIMA, 1979), embora redução na síntese de proteína microbiana (PETRI et al., 1988) e ineficiente degradação da fibra (KOMISARCZUK et al., 1987) tenham sido descritas.

Se a conversão alimentar ( $\mathrm{kg}$ matéria seca ingerida/ kg ganho de peso) for relacionada ao nível de $\mathrm{P}$ na dieta , obtém-se a seguinte equação:

Conversão alimentar $=19,9-0,808 \mathrm{P},\left(\mathrm{r}^{2}=0,79\right)$

em que $\mathrm{P}=\mathrm{g} \mathrm{P} / \mathrm{dia}$

ANOVA: $\mathrm{P}<0,001$

Esta equação indica menor eficiência de utilização do alimento durante a deficiência de P, também descrita por GARTNER et al.(1982), dando suporte à tese de interferência no metabolismo intermediário. A deficiência de $\mathrm{P}$ tem sido relacionada à queda dos teores de $\mathrm{P}$ nos tecidos moles. Esta queda levaria à redução da taxa metabólica, devido à menor disponibilidade de AMP cíclico ou outras moléculas de ácidos nucléicos (Clarke, 1990, citado por TERNOUTH, 1991) e/ou à acumulação de metabólitos intermediários causada pela redução no metabolismo intracelular (MILTON e TERNOUTH, 1985).

Conclui-se, então, que 11 e 15 g P/dia proporcionam resultados semelhantes em relação à digestibilidade, ao consumo e à utilização de matéria seca e ganho de peso. Como foi comentado anteri- 
ormente, os ganhos de peso dos animais dos tratamentos 1 e 2 foram semelhantes, tendo sido superiores àqueles observados em novilhos do tratamento 3 (Figura 1). Os tratamentos 1 e 3 mostraram diferenças significativas $(\mathrm{P}<0,01)$ quanto ao peso vivo já aos 71 dias de experimentação.

Neste estudo, os requisitos de P para novilhos Nelore com cerca de $300 \mathrm{~kg}$ de peso vivo e ganhando entre 500-600 g/dia situaram-se acima de $5 \mathrm{~g} / \mathrm{dia}$, sendo próximos ou inferiores a $11 \mathrm{~g} / \mathrm{dia}$. Os requisitos de $\mathrm{P}$ sugeridos por autoridades de diferentes países variaram de cerca de 11 (AGRICULTURAL AND FOOD RESEARCH COUNCIL - AFRC, 1991), 12 (NRC, 1996), 13 (TERNOUTH et al., 1996) até os 14 g/dia (NRC, 1984). É interessante notar que este valor do AFRC (1991) foi calculado para dietas à base de concentrados e subiria para cerca de $17 \mathrm{~g} /$ dia, se corrigida para dietas contendo mais de $50 \%$ de forrageiras.

Os requisitos nutricionais são calculados a partir das perdas endógenas, dos requisitos líquidos para retenção nos tecidos e do coeficiente de absorção. Existem divergências entre os valores usados nos cálculos feitos pelo NRC (1996) e AFRC (1991), especialmente quanto ao coeficiente de absorção utilizado. Enquanto o AFRC (1991) recomenda os fatores 0,58 e 0,70 para dietas à base de forrageiras e concentrados, respectivamente, o NRC (1996) utiliza o fator 0,68 para ambas. Pesquisadores australianos (TERNOUTH et al., 1996) sugeriram o valor de 0,75 para o coeficiente de absorção de $\mathrm{P}$ de bovinos em pastagens tropicais, observando que $\mathrm{O}$ animal aparentemente se adapta a dietas baixas em $\mathrm{P}$, aumentando seu coeficiente de absorção para até 0,90 (COATES e TERNOUTH, 1992). Cabe ressaltar que esses autores observaram ganhos de peso anuais em torno de $152 \mathrm{~kg}$ em novilhas (peso médio de $250 \mathrm{~kg}$ ) mantidas em forrageiras tropicais e consumindo não mais que $6 \mathrm{~g}$ P/dia, sem alteração significativa das reservas ósseas. Nenhuma das estimativas mencionadas se aproximam deste valor, mostrando que a definição dos requisitos mínimos de $\mathrm{P}$ continua sendo um desafio, dadas as interações entre nutrientes e a capacidade do osso de funcionar como reserva de $\mathrm{P}$.

\section{Conclusões}

Nas condições deste estudo, $11 \mathrm{~g} /$ dia de fósforo foram suficientes para propiciar ganhos da ordem de $500 \mathrm{~g} /$ dia a novilhos Nelore com cerca de $300 \mathrm{~kg}$ de peso vivo. A deficiência de $\mathrm{P}$ não comprometeu consistentemente a digestibilidade in vivo da matéria seca e da matéria orgânica, mas o consumo, o ganho de peso vivo e a conversão alimentar foram negativamente afetados.

\section{Referências Bibliográficas}

AGRICULTURAL AND FOOD RESEARCHCOUNCIL - AFRC. 1991. Technical Committee on Responses to Nutrients. Commonwealth Agricultural Bureaux International. A reappraisal of the calcium and phosphorus requirements of sheep and cattle. Nut. Abst. and Rer. (Series B), 61 (9):573-612.

BORTOLUSSI, G., TERNOUTH, J.H., McMENIMAN, N.P. 1996. Dietary nitrogen and phosphorus depletion in cattle and their effects on live weight gain, blood metabolite concentrations and phosphorus kinetics. J. Agric. Sci., 126(4):493-501.

CALL, J.W., BUTCHER, J.E., BLAKE, J.T. et al. 1978. Phosphorus influence on growth and reproduction of beef cattle. J. Anim. Sci., 47(1):216-225.

CALL, J.W., BUTCHER, J.E., SHUPE, J.L. et al. 1986. Dietary phosphorus for beef cows. Am. J. Vet. Res., 47(2):475-81.

COATES, D.B., TERNOUTH, J.H. 1992. Phosphorus kinetics of cattle grazing tropical pastures and implications for the estimation of their phosphorus requirements. J. Agric. Sci., 119(3):401-409.

DURAND, M., KAWASHIMA, R. 1979. Influence of minerals in rumen microbial digestion. In: RUCKSBUSH, Y., THIVEND, P. (Eds.) Digestive physiology and metabolism in ruminants. Lancaster: MTP Press. p.375-408.

EZEQUIEL, J.M.B., EZEQUIEL, P.A., COELHO DA SILVA, J.F. et al. Exigências de cálcio e de fósforo de bovideos: frações endógenas. In: REUNIÃO ANUAL DA SOCIEDADE BRASILEIRA DE ZOOTECNIA, 25, 1988, Viçosa. Anais... Viçosa: SBZ, 1988. p.84.

GARTNER, R.J.W., MURPHY, G.M., HOEY, W.A. 1982. Effects of induced, subclinical phosphorus deficiency on feed intake and growth of beef heifers. J. Agric. Sci., 98(1):23-29.

HARRIS, L.E. 1970. Os métodos químicos e biológicos empregados na análise de alimentos. Gainesville: University of Florida. Paginação descontínua.

KOMISARCZUK, S., MERRY, R.J., McALLAN, A.B. 1987. Effect of different levels of phosphorus on rumen microbial fermentation and synthesis determined using a continuous culture technique. Br. J. Nutr., 57(2):279-290.

LITTLE, D.A. 1980. Observations on the phosphorus requirement of cattle for growth. Res. Vet. Sci., 28(2):258-260.

MARGON, A.L., COELHO DA SILVA, J.F., CASTRO, A.C.G. et al. 1982. Composição corporal de macrominerais (Ca, P, $\mathrm{Mg}$, Na e K) para engorda de novilhos zebu. R. Soc. Bras. Zootec., 11(2):237-51.

MILTON, J.T.B., TERNOUTH, J.H. 1985. Phosphorus metabolism in ruminants. II. Effects of inorganic phosphorus concentration upon food intake and digestibility. Aust. J. Agric. Res., 36(4):647-654.

MORAES, J.F.V., RABELO, N.A. 1986. Um método simples para a digestão de amostras de plantas. Goiânia: EMBRAPACNPAF. 12p. (EMBRAPA-CNPAF. Documentos, 12).

NEL, J.W., MOIR, R.J. 1974. The effect of ruminal and duodenal application of different levels of calcium and phosphorus to sheep on semi-purified diets. S. Afr. J. Anim. Sci., 4:1-20.

NATIONAL RESEARCH COUNCIL - NRC. 1984. Nutrient 
Rev. bras. zootec.

requirements of beef cattle. 6.ed.rev., Washington: National Academy Press. 90p.

NATIONAL RESEARCH COUNCIL - NRC. 1996. Nutrient requirements of beef cattle. 7.ed.rev., Washington: National Academy Press. 242p.

PETRI, A., MUENSCHEN, H., BREVES, G. et al. 1988. Response of lactating goats to low phosphorus intake. 2. Nitrogen transfer from rumen ammonia to rumen microbes and proportion of milk protein derived from microbial amino acids. J. Agric. Sci., 111:265-271.

SAS Institute (Cary, USA). 1985. Statistical analysis system user's guide: basics. Cary. 1290p.

TERNOUTH, J.H. 1991. The kinetics and requirements of phosphorus in ruminants. Recent advances on the nutrition of herbivores, 15:143-151. Separata.

TERNOUTH, J.C., SEVILLA, C.C. 1990. The effects of low levels of dietary phosphorus upon the dry matter intake and metabolism of lambs. Aust. J. Agric. Res., 41(1):175-184.
1195

TERNOUTH, J.H., BORTOLUSSI, G., COATES, D.B. et al. 1996. The phosphorus requirements of growing cattle consuming forage diets. J. Agric. Sci., 126(4):503-510.

TOKARNIA, C.H., DOBEREINER, J., MORAES, S.S. 1988. Situação atual e perspectivas da investigação sobre nutrição mineral em bovinos no Brasil. Pesq. Vet. Bras., 8(1-2):1-16. UNDERWOOD, E.J. 1981. The mineral nutrition of livestock. 2.ed. Farnham Royal: CAB. p.102-103.

Recebido em: 23/04/1998

Aceito em: 09/11/1999 